\title{
On Preparations and Applications of Nano-materials for Batteries
}

\author{
Dingzhu Yang \\ School of Materials Science \& Engineering, University of Science \& Technology Beijing, Beijing, \\ 100083, China
}

Keywords: Nanotechnology, Battery materials, Preparations and applications

\begin{abstract}
Nanotechnology is the rapid developing. The nanotechnology in battery materials can enhance the storage capacity of a battery, reduce swelling and loss of the battery and improve use efficiency, which is of positive significance for the electrical equipment. Based on the analysis of current development of nano battery, the preparations of several nano materials for battery are discussed, which can be better applied in the production of life and point out the direction for the development of modern nano battery technology.
\end{abstract}

\section{Current Development Situation of Nano-materials for Batteries}

Introduction to Nano-battery. The nano battery is a battery made of nanometer technology, and the size of the material is less than $100 \mathrm{~nm}$. These cells are of nanometer size or can be combined together as a large cell such as a nano pore cell. Lithium ion battery technology using traditional active materials. The size of cobalt oxide or manganese oxide ranges from 5 to 20 microns. Many disadvantages of Nano Engineering can improve the existing battery technology, such as low power density and volume expansion. The chemical energy can be converted into electrical energy, which is composed of three parts: anode (positive electrode), cathode (negative electrode) and electrolyte. The anode and cathode have two different chemical potentials, depending on the reaction at the two end. The electrolyte can be either a solid or a liquid, a dry material or a wet material, respectively. The boundary between the electrode and the electrolyte is called the electrolyte phase, and the chemical energy stored in the cell is converted to electrical energy by the voltage applied to the electrode.

Shortages of Traditional Battery. The ability of the battery to store electricity depends on its energy density and power density, and the cycle and volume expansion are also important considerations. In order to improve the battery technology, it is necessary to maximize the cycle capacity and energy and power density, and the volume expansion must be minimized. During lithium insertion, the volume of the electrode expands, causing mechanical strain. Mechanical strain damages the structural integrity of the electrode, leading to fracture. In the current lithium ion battery technology, lithium diffusion rate is slower. When the volume expansion of the nanoparticles is smaller than that of the total volume of the cell, the nano particles can reduce the mechanical strain on the material when the battery goes through the cycle. The small volume expansion associated with the nanoparticles also improves the reversibility of the battery, and the battery undergoes many cycles without loss of charge.

\section{Preparations of Nano-materials for Batteries}

Preparation of Nano- $\mathrm{MnO}_{2}$. Naturally occurring manganese dioxide contains impurities and a large amount of manganese oxide (III), only a limited number of sediments contain sufficient purity characteristics for the battery industry. The manganese dioxide needs high purity manganese dioxide, the battery needs the electrolytic manganese dioxide, and the ferrite needs the chemical nanometer manganese dioxide. The chemical preparation method of nano manganese dioxide from natural manganese dioxide, and the use of nitrogen tetroxide and water into nitrate (II) solution. Evaporation of water leaving crystalline nitrate. At a temperature of 400 DEG C, the salts decompose, release $\mathrm{N} 2 \mathrm{O} 4$, and leave the residue of purified mno2. EMD is commonly used in zinc manganese dioxide rechargeable alkaline (Zn RAM) batteries. For these applications, purity is very important. EMD is 
produced in a manner similar to that of electrolytic tough pitch (ETP) copper, which is dissolved in sulfuric acid, sometimes mixed with manganese sulfate, and transferred between two electrodes. Nano $\mathrm{MnO}_{2}$ was dissolved as sulfate into the solution and deposited on the anode. Nano manganese hydroxide (II) has two well characterized polymorphs alpha and beta. The alpha structure is composed of a $\mathrm{Mn}(\mathrm{OH})_{2}$ layer with an intercalated anion or water, and the $\mathrm{Mn}^{2+}$ and $\mathrm{OH}^{-}$ions are used in the beta type close packed structure. In the water presence situation, alpha polymorphs are usually recrystallized in the form of beta. Some kinds of gamma manganese hydroxide are found with the feature of larger range of crystal structures.

Preparation of Nano-graphene. Graphene has been widely used in electrochemical systems since the first time in 2004. Graphene provides high surface area and good conductivity, the lithium ion battery technology at present, graphite inhibited the smooth two-dimensional network of lithium ion insertion, lithium ion must be around 2D to reach the graphite sheet traveling electrolyte, charging speed will slow down the battery, is currently in the research of porous graphene materials to improve the problem. Porous graphene involves the formation of defects in 2D sheets or the creation of porous structures based on 3D graphene. As the anode, graphene will provide the expansion of the space, so as not to cause the problem of volume expansion. 3D graphene shows a very high extraction rate of lithium ions, indicating a large reversible capacity. Similarly, the random distribution of ions seen below the graphene anode will allow the lithium ion to be stored not only on the inner surface of the graphene, but also on the nanopore between the graphene layers. Carbon based Si based nanostructures can be produced, nanostructured materials disposed on carbon based substrates, and nano structures comprising nanoscale scaffolds. Graphene has a very large irreversible mechanism in the first step of the lithium. Because of the large surface area of graphene, graphene has a large initial irreversibility. The research of anode graphene is still in progress, and carbon nanotubes have been used as the electrode to insert the battery to improve the capacity of the battery.

\section{Applications of Nano-materials for Batteries}

Lithium Ion Battery. In the 90s of last century, the application of nano materials in lithium ion batteries has made great progress. For example, nano materials and nano composite materials can be used as lithium intercalation materials, which can improve the reversible lithium insertion capacity and cycle life. The characteristics of active nano material with large surface area, lithium ion insertion and extraction depth of small, short stroke, the electrode at high current charge and discharge the degree of polarization is small, high reversible capacity, long cycle life; nano materials of high pore ratio for migration of organic solvent molecules provides free space, so that the organic solvent has good compatibility, but also provides a lot of space for lithium ion insertion and extraction, further improve the lithium capacity and energy density. Nano carbon materials and nano two tin oxide materials are mainly used as anode materials for lithium ion batteries. In addition, the catalytic group of Hollandite type $\mathrm{MnO}_{2}$ nano fiber can also be used as a fuel cell. The cathode material nano crystalline VO2 can be used as a $4 \mathrm{~V}$ lithium-ion battery, but also can be used as an ode to combine $\mathrm{LiMn}_{2} \mathrm{O}_{4}$ to form1.5V lithium ion batteries.

Solar Battery. China Institute of plasma physics on dye-sensitized solar cells for many patents, patents were related to electrolyte, electrode preparation method, dye sensitized solar cell sealing method, electrolyte solution with CN1444290 open dye-sensitized nano thin film solar cell, A, B or B $\mathrm{F}, \mathrm{A}, \mathrm{B}$, or $\mathrm{F}$ as the main component, through the compound or compound one or several components of the other four components in the composition of the electrolyte solution, the A component of the organic solvent or mixed solvent; the B component of I2/I- reversible electrochemical oxidation reduction (I3-/I-) the electric components with agent C; light anode; D component iodide in cation complexing agent; compounding agent E component I2 F component; ionic liquid; G components of UV absorbers. The electrolyte solution has the advantages of high conductivity, low viscosity, good electrochemical reversibility, good low temperature stability and strong ultraviolet resistance. Chinese Institute of plasma physics discloses CN2724205 large area internal parallel dye-sensitized 
solar cells, including the upper and lower surfaces of a transparent substrate, a transparent conductive film on a transparent substrate, a conductive electrode and a catalyst layer is arranged on the transparent conductive film, a transparent conductive film on the conductive electrode with nano porous semiconductor materials block interval arrangement, nano porous semiconductor material impregnated in dye. The two transparent substrates are stacked together, and the periphery is sealed into a cavity. The utility model makes a parallel electrode in the battery to obtain the required output current of the solar cell. Battery sealing function, to ensure the long-term stability of the battery operation. The utility model has the advantages of simple and easy operation, low cost and stable battery performance.

Alkaline $\mathbf{Z n} / \mathbf{M n O}_{2}$ Battery. Nano $\mathrm{MnO}_{2}$ is synthesized by sol-gel method, microemulsion method, solid-state reaction method to synthesize, through scientific and effective mixed with EMD, can greatly enhance the purity of nano gamma $-\mathrm{MnO}_{2}$, which has important meaning for the manufacture of alkaline zinc manganese battery. $\mathrm{Bi}$ doped nano $\mathrm{MnO}_{2}$. The modified $\mathrm{MnO}_{2}$ was synthesized by the addition of $\mathrm{Bi}_{2} \mathrm{O}_{3}$, and the discharge capacity was improved to some extent by the method of mixing $\mathrm{Bi}-\mathrm{MnO}_{2}$ with nanometer and micron grade. The co reduction and $\mathrm{CO}$ oxidation of $\mathrm{Bi}-\mathrm{Mn}$ complexes formed by a series of different valence states of $\mathrm{Bi}$ during the charge discharge process can effectively inhibit the formation of $\mathrm{Mn}_{3} \mathrm{O}_{4}$, which can greatly improve the charging ability of the electrode. A small amount of $\mathrm{ZnO}$ was added into the alkaline manganese dioxide battery in order to suppress the self-discharge of zinc anode in the electrolyte. The more uniform dispersion of $\mathrm{ZnO}$ in the liquid, the more favorable to control self-discharge. Nano $\mathrm{ZnO}$ has been used in medicine and so on. The mercury free alkali manganese battery towards development, using nano $\mathrm{ZnO}$ is one of the alternative methods. The key is to pay attention to the surface modification of nano $\mathrm{ZnO}$ materials. Nanoscale $\mathrm{In}_{2} \mathrm{O}_{3}$. $\mathrm{In}_{2} \mathrm{O}_{3}$ is one of the choice of alkaline manganese battery inorganic mercury substituting inhibitor, has been developed and produced with high purity nano $\mathrm{In}_{2} \mathrm{O}_{3}$ mercury free alkali manganese battery, the material has large surface area, good dispersion characteristics, corrosion inhibition effect is better. It is applied to the mercury free alkaline manganese battery has good suppression the effect of gas.

\section{Development Prospect of Nano-materials for Batteries}

The use of nanotechnology to produce a battery has the following advantages: increasing the available power of the battery, reducing the time required for charging the battery. This is achieved by coating the surface of the electrode with nanoparticles, which increases the surface area of the electrode, allowing more current to flow between the electrode and the chemical inside the cell. When the battery is not in use, the Nano material can be used as a coating for separating the electrode from the liquid in the battery. In the current battery technology, liquid and solid interaction, resulting in low level discharge, which reduces the service life of the battery. There are some problems in the application of nanotechnology in the cell, such as low density and high surface area. The larger the surface area is, the easier the oxidation reaction occurs on the surface of the air. Due to the low density of the nanoparticles, there is a high resistance between the particles, which reduces the conductivity of the material. Nano materials difficult to manufacture, increase costs. Although nanomaterials may greatly enhance the capacity of the battery, but they may be costly.

With the development of nano battery for light electric equipment has an important role, this paper based on the nano battery material on the preparation and application of analysis, through the analysis of nano battery material preparation program and production life application. Its preparation can be summarized and elucidated. Its application is discussed. With the development of nano battery technology, battery production technology in China will continue to progress. It can also be seen that the traditional battery materials are no longer suitable for the needs of a variety of battery production. The replacement of nano battery materials is a bright and tortuous road, there will be many difficulties and challenges in this way. This task is long and arduous, it is necessary to combine the actual production experience, and constantly summarize. In order to achieve their own long-term 
development and bold innovation, we should make use of innovative thinking for modernization to move towards the production goal of efficient energy saving battery.

\section{References}

[1] Jiang Keyu, Zhao Zhenjie, Yang Xiehong, Yang Jiexin, Developing nanostructure materials [J]. Physics Experimentation, 2001, 21(7): 5-7.

[2] Fu Zhijia, New progress in application of nano- $\mathrm{TiO}_{2}$ in solar cells [J]. Journal of Baoji University of Arts and Sciences (Natural Science), 2010, 30(3): 47-52.

[3] Tian Chunxia, Recent Progress of Nano-Scale Material Applied in Lithium Ion Batteries [J]. Chinese Journal of Rare Metal, 2002, 26(5): 397-400.

[4] She Jianfang, Present Status and Development of Nanostructured Materials [J]. Rare Metals and Cemented Carbides, 2001(5): 42-45.

[5] Yingyue Zhang, Evan Mintzer, and Kathryn E. Uhrich, Synthesis and Characterization of PEGylated Bolaamphiphiles with Enhanced Retention in Liposomes [J]. Journal of Colloid and Interface Science, 2016, 482, 19-26.

[6] Dalia S. Abdelhamid, Yingyue Zhang, Daniel R. Lewis, Prabhas V. Moghe, William J. Welsh, and Kathryn E. Uhrich, Tartaric Acid-based Amphiphilic Macromolecules with Ether Linkages Exhibit Enhanced Repression of Oxidized Low Density Lipoprotein Uptake [J]. Biomaterials, 2015, 53, 32-39. 